\title{
EQUITABLE DISTRIBUTION OF INDIVISIBLE OBJECTS
}

\author{
Stephen DEMKO and Theodore P. HILL \\ School of Mathematics, Georgia Institute of Technology, Atlanta, GA 30332, U.S.A.
}

\begin{abstract}
Deterministic and randomized solutions are developed for the problem of equitably distributing $m$ indivisible objects to $n$ people (whose values may differ), without the use of outside judges or side-payments. Several general bounds for the minimal share are found; a practical method is given for determining an optimal lottery and the largest minimal share; and the case of repeated allocations is analyzed.
\end{abstract}

Key words: fair division problems; indivisible objects; lottery; fair lottery; independent lottery; deterministic distribution; randomized allocation; optimal share.

\section{Introduction}

Suppose $m$ indivisible objects (or tasks) must be distributed to $n$ people whose relative and total values of the objects may differ, as is often the case in estate settlements or committee assignments. Is it possible to distribute the objects in such a way that each person receives what he considers a fair share, or in some other 'optimal' way?

Classical solutions of such problems usually involve selling the objects and distributing the money, employment of an outside judge or assessor, or use of side-payments (see e.g., Steinhaus, 1948, 1949, 1960; and Dubins, 1977). Selling the objects and distributing the money, however, essentially ignores both the individual values of the participants and the indivisibility of the objects (which are in effect replaced by the single completely-divisible object 'money'); employment of an outside judge or assessor introduces a new value judgement not inherent in the original problem and often not closely related to the original participants' values; and use of sidepayments assumes the existence of a new completely-divisible object (money), also not inherent in the original problem and which often is not available (in sufficient quantity) to all the participants. The objective of this paper is to study several solutions of the distribution problem which stay within the framework of the original 
problem, that is, which do not employ outside judges or side-payments.

It is assumed that each participant is to share equally in the estate, and that each of the values of the participants is known (i.e., there is complete information) and is normalized with total value unity, so that the entire problem in the case of $n$ people and $m$ objects is completely described by a single $n \times m$ row stochastic matrix $A=\left(a_{i j}\right)$, where $a_{i j}$ represents the value of the $j$ th object to the $i$ th person.

The assumption of normalization to unity simply reflects the use of relative (as opposed to absolute) values of the objects in determining utilities. In other contexts, such as those where side-payments or arbitration are allowed (see e.g., Kalai, 1985; or Steinhaus, 1960), absolute values of the objects may well be important in determining the solution or side-payment. But when no side-payments or arbitration are allowed, relative values of the objects seems to be the only important factor. If a participant considers a particular collection of objects to be worth exactly one-third the total estate, then he values this collection equally to any other collection worth exactly one-third to him, and values it more than any collection he feels is worth strictly less than one-third, regardless of the absolute values of each of the objects. (This is equivalent to an assumption of 'individual scale-invariance', where for convenience all total values are simply rescaled or renormalized to unity.)

It should be emphasized that the treatment of the distribution problem in this paper is measure-theoretic and probabilistic, not game theoretic or economic, and notions such as use of coalitions, bargaining or deception are not addressed. The objective studied here is to maximize the minimum share received by the participants, and a solution which is optimal in this sense is clearly Pareto optimal (e.g., see Kalai, 1985). When risks or lotteries are involved, an expected-value criterion assuming risk neutrality is used.

Example 1. (Several value-matrices for $n=m=2$.)

$$
A_{1}=\left(\begin{array}{cc}
0.7 & 0.3 \\
0.4 & 0.6
\end{array}\right) ; \quad A_{2}=\left(\begin{array}{cc}
0.7 & 0.3 \\
0.7 & 0.3
\end{array}\right) ; \quad A_{3}=\left(\begin{array}{ll}
0.7 & 0.3 \\
0.6 & 0.4
\end{array}\right) \text {. }
$$

For $A_{1}$, an obvious solution is to give (deterministically) object 1 to person 1 and object 2 to person 2 ; this certainly guarantees that each participant receives at least half the value of the total estate according to his own preferences. For $A_{2}$, no such deterministic solution is fair since one of the two participants must receive a share he considers $\leq 0.3$, but an obvious randomized solution is to toss a fair coin and give object 1 to person 1 and object 2 to person 2 if the coin shows 'heads' (and vice versa otherwise); this guarantees each person at least 0.3 of the estate, and an expected share of half the estate. For $A_{3}$, the coin-tossing solution is also fair, but an optimal randomized solution (see Example 4.3 below) is to give object 2 to person 2 deterministically, and also give object 1 to person 2 with probability $\frac{3}{13}$, yielding an expected share of $\frac{7}{13}$ to each person.

This paper is organized as follows: Section 2 contains an analysis of deterministic solutions including a proof of the NP-completeness of the problem of deciding the 
existence of fair deterministic distributions, and a lower bound on the largest possible minimal share as a function of the maximum entry of the value matrix; Section 3 introduces the notion of randomized solutions and proves the existence of optimal 'fair' and 'independent' lotteries; Section 4 contains methods for practical calculation of optimal lotteries and minimal shares (essentially by reduction to a linear programming problem) and identifies some general bounds for optimal randomized distributions; and Section 5 contains an analysis of the problem of repeated (or periodic) distribution of the same objects to the same people over time.

\section{Deterministic solutions}

Throughout this paper $S_{n, m}$ is the set of $n \times m$ row stochastic matrices and $D_{n, m}$ is the subset of $S_{n, m}$ consisting of matrices whose only entries are 0 or $1 .(M)_{i j}$ is the $i j$ th entry of the matrix $M$.

Definition 2.1. The deterministic-distribution value of $A \in S_{n, m}, V_{\mathrm{D}}(A)$, is given by

$$
V_{\mathrm{D}}(A)=\max \left\{\min _{i \leq n}(A Q)_{i i}: Q \in D_{m, n}\right\} .
$$

(That is, if the objective is to distribute the objects deterministically so as to make the minimum share as large as possible, $V_{\mathrm{D}}(A)$ is the largest possible minimum share.)

The problem of deciding whether a given problem (matrix) has a deterministic 'fair' solution (i.e., one with $V_{\mathrm{D}}(A) \geq 1 / n$ ) may be computationally intractable. It is at least NP-complete as is now demonstrated (so there is no known polynomial (in the variables $n, m$ ) time algorithm which will decide if there is a fair solution).

Consider the subclass of problems which involve only 2 people who assign the same rational values to each object. That is, $(A)_{1 j}=(A)_{2 j}=p_{j} / N$ for some integers $p_{j}, N$, with $\sum p_{j}=N$. If there are $m$ objects, there will be a fair deterministic solution if and only if there is a subset $S \subseteq\{1,2, \ldots, m\}$ with $\sum_{j \in S} p_{j}=\sum_{j \notin S} p_{j}$. The problem of determining if there is such a subset is the partition problem - one of the six basic NP-complete problems of Garey and Johnson (1979). This proves the following result.

Theorem 2.2. The problem of determining whether fair deterministic solutions exist (i.e., whether $\left.V_{\mathrm{D}}(A) \geq 1 / n\right)$ is at least NP-complete.

On the other hand, for the class of problems described above it is not hard to construct random distributions which are fair in the sense that each person's expected share is 0.5 . Consider the case $n=2$ where each person assigns the same values to $m \geq 2$ objects; then at least $m-1$ of them can be distributed deterministically. For the 2 person case, let the values of the objects be $v_{1} \leq v_{2} \leq \ldots \leq v_{m}$. Distribute the 
objects one at a time as follows: person 1 gets $v_{1}, v_{3}, \ldots$, person 2 gets $v_{2}, v_{4}, \ldots$, and stop distributing when one person would have more than 0.5 on his next turn. This implies $v_{1}+v_{3}+\ldots+v_{2 k-1} \leq 0.5$ and either $v_{2}+\ldots+v_{2 k-2} \leq 0.5$ or $v_{2}+\ldots+v_{2 k} \leq 0.5$ with the next allocation being the one that would raise one of the person's share to more than 0.5 . The claim is that there is only one object remaining. For if at least two objects remained, their values, $v_{j} \leq v_{j+1} \ldots \leq v_{m}$, would raise both parties' worth to more than $\frac{1}{2}$ contradicting the assumption that $v_{1}+\ldots+v_{m}=1$. It is then easy to arrange a lottery for the one remaining object that will insure both parties of an expected value of 0.5 . A short calculation shows that if person 1 has a share of $\alpha$ and person 2 has a share of $\beta$ after all $m-1$ pieces have been distributed, then assigning the $m$ th piece to person 1 with probability $\left(\beta-\alpha+v_{m}\right) / 2 v_{m}$ gives each person an expected share of 0.5 .

The next theorem gives a sharp lower bound for the largest possible minimum share $V_{\mathrm{D}}(A)$ as a function of the number of people and the largest entry of $A$.

Definition 2.3. (Hill, 1987a). $V_{n}:[0,1] \rightarrow\left[0, n^{-1}\right]$ is the unique non-increasing function satisfying

$$
V_{n}(\alpha)=1-k(n-1) \alpha \quad \text { for } \quad \alpha \in\left[(k+1)[k((k+1) n-1)]^{-1},(k n-1)^{-1}\right]
$$

for all $k \geq 1$.

Theorem 2.4. If $A=\left(a_{i j}\right) \in S_{n, m}$ satisfies $a_{i j} \leq \alpha$ for all $i \leq n$ and $j \leq m$, then $V_{\mathrm{D}}(A) \geq V_{n}(\alpha)$. Moreover, this bound is sharp; for each $n \geq 1$ and $\alpha \in(0,1]$ there is an $m \geq 1$ and an $A \in S_{n, m}$ with $a_{i j} \leq \alpha$ for all $i$ and $j$, and $V_{\mathrm{D}}(A)=V_{n}(\alpha)$.

Proof. The first part of the conclusion is a special case of the following theorem of Hill (1987a). If $\mu_{1}, \ldots, \mu_{n}$ are probability measures on the same measurable space $(X, \mathscr{F})$, and if $\mu_{i}(A) \leq \alpha$ for each $\mu_{i}$-atom $A$ and each $i$, then there is a measurable partition $\left\{B_{i}\right\}_{i=1}^{n}$ of $X$ so that $\mu_{i}\left(B_{i}\right) \geq V_{n}(\alpha)$ for all $i=1, \ldots, n$. That the bound is attained for all $n \geq 1$ and all $\alpha \in(0,1]$ is seen by the following analog of an example of Hill (1987a). Fix $\alpha>0$, let $k$ be such that $\alpha \in\left[(k+1)[k((k+1) n-1)]^{-1}\right.$, $(k n-1)^{-1}$ ], and let $m=k n$ and $A \in S_{n, m}$ be the matrix given by $a_{i j}=\alpha$ for all $i \leq n$ and all $j<m$, and $a_{i m}=1-\alpha(m-1)$ for all $i \leq n$. It is easy to check that $V_{\mathrm{D}}(A)=1-k(n-1) \alpha$. .

Example 2.5. Three people must divide an estate of $m$ objects, none of which is valued at more than $10 \%$ of the total estate by any person. Then there is always a way of assigning objects to people so that each person receives a share he values at least $V_{3}\left(\frac{1}{10}\right)=\frac{3}{11}$ of the total estate, and in general it is not possible to do better.

\section{Randomized solutions}

The purpose of this section is to introduce and investigate the notion of distribut- 
ing $m$ indivisible objects to $n$ people randomly, for example through the use of a lottery. Such solutions in general may (with positive probability) assign none of the objects to a particular person; however, it is the expected share of each participant that is compared, the tacit assumption being that a person is risk neutral, e.g., each person would value equally a lump sum of $\$ 500$ and a 'fifty-fifty' chance at $\$ 1000$ (with probability $\frac{1}{2}$ he wins $\$ 1000$, and with equal probability he wins nothing), and would prefer either of these over any option with expected value strictly less than $\$ 500$. Problems of risk-averting or risk-seeking values (or nonlinear utilities) are not addressed in this paper.

Definition 3.1. A lottery $L$ on $(n, m)$ is a probability measure on $D_{m, n}$. (The probability person $i^{\prime}$ gets object $j^{\prime}$ under lottery $L, P_{L}\left(j^{\prime} \rightarrow i^{\prime}\right)$, is

$$
\left.P_{L}\left(j^{\prime} \rightarrow i^{\prime}\right)=\sum\left\{d_{j^{\prime} i^{\prime}}, L(D): \quad D=\left(d_{i j}\right) \in D_{m, n}\right\} .\right)
$$

The set of all lotteries on $(n, m)$ will be denoted by $\mathscr{L}(n, m)$.

Example 3.2. (a) Roll an ' $n$-die' once; if ' $i$ ' shows, then person $i$ gets all $m$ objects. This corresponds to the lottery $L_{1} \in \mathscr{L}(n, m)$ with $L_{1}(D)=1 / n$ if $D$ has one column all 1 's, and $L_{1}(D)=0$ otherwise.

(b) Roll an ' $n$-die' $m$ times (independently); if ' $i$ ' shows on the $j$ th roll, then person $i$ gets object $j$. This corresponds to the lottery $L_{2}$ with $L_{2}(D)=n^{-m}$ for all $D \in D_{m, n}$.

(c) Flip a coin once; if 'head' shows, person 1 gets all $m$ objects, otherwise person 2 gets all $m$ objects. This corresponds to the lottery $L_{3}$ with $L_{3}\left(D_{1}\right)=L_{3}\left(D_{2}\right)=\frac{1}{2}$, where $D_{1}$ is the stochastic matrix with first column identically 1 , and $D_{2}$ with the second column identically 1 .

Definition 3.3. The $A$-value to person $i$ of lottery $L, V(A, L, i)$, is

$$
V(A, L, i)=\sum_{j=1}^{m} a_{i j} P_{L}(j \rightarrow i) ;
$$

the (minimum) $A$-value of lottery $L, V(A, L)$, is

$$
V(A, L)=\min _{i \leq n} V(A, L, i) \text {; and }
$$

the randomized value of $A, V_{\mathrm{R}}(A)$, is

$$
V_{\mathrm{R}}(A)=\sup \{V(A, L): L \in \mathscr{L}(n, m)\} .
$$

\section{Example 3.4.}

(a) Let $L_{1}, L_{2}, L_{3}$ be as in Example 3.2. Then for all $A \in S_{n m}$ :

$$
\begin{gathered}
V\left(A, L_{1}, i\right)=V\left(A, L_{2}, i\right)=1 / n \text { for all } i \leq n \text { and } \\
V\left(A, L_{1}\right)=V\left(A, L_{2}\right)=1 / n ;
\end{gathered}
$$


and

$$
\begin{aligned}
& V\left(A, L_{3}, 1\right)=V\left(A, L_{3}, 2\right)=\frac{1}{2} \text { and } \\
& V\left(A, L_{3}, i\right)=V\left(A, L_{3}\right)=0 \text { for all } i>2 \text { if } n>2 . \\
& V_{\mathrm{R}}\left(\begin{array}{ll}
1 & 0 \\
0 & 1
\end{array}\right)=1 ; \quad V_{\mathrm{R}}\left(\begin{array}{ll}
1 & 0 \\
1 & 0
\end{array}\right)=\frac{1}{2} .
\end{aligned}
$$

(b)

Definition 3.5. Lottery $L$ is independent if

$$
\begin{aligned}
& P_{L}\left(j_{k} \rightarrow i_{k} \text { for all } k=1, \ldots, r\right)=\prod_{k=1}^{r} P_{L}\left(j_{k} \rightarrow i_{k}\right) \\
& \quad \text { for all } r \leq m, \text { all subsets }\left\{j_{1}, \ldots, j_{r}\right\} \text { of }\{1, \ldots, m\}, \\
& \quad \text { and all } i_{k} \in\{1, \ldots, n\}, k=1, \ldots, r ;
\end{aligned}
$$

otherwise $L$ is dependent.

(Intuitively, an independent lottery is one in which there is a separate (and independent) sublottery for each object.)

Example 3.6. Let $L_{1}, L_{2}, L_{3}$ be as in Example 3.2. Then $L_{1}$ is dependent, $L_{2}$ is independent, and $L_{3}$ is dependent.

Theorem 3.7. Independent lotteries suffice. That is, for all $A \in S_{n, m}$ there is a $Q \in S_{m, n}$ with

$$
V(A, Q)=V_{\mathrm{R}}(A)
$$

Proof. That the supremum is attained by some lottery $L^{*}$ follows easily from the compactness of the space of probabilities on the finite set $D_{m, n}$ and the continuity in $A$ of $V_{\mathrm{R}}(A)$. Let $Q \in S_{m, n}$ denote the independent lottery defined by $q_{i j}=$ $P_{L^{*}}(j \rightarrow i)$ for $l \leq j \leq m, 1 \leq i \leq n$. Then $V\left(A, L^{*}, i\right)=\sum_{j=1}^{m} a_{i j} P_{L^{*}}(j \rightarrow i)=\sum_{j=1}^{m}$ $a_{i j} q_{j i}=(A Q)_{i i}=V(A, Q, i)$.

Definition 3.8. Lottery $L$ is fair for $A$ if $V(A, L) \geq 1 / n$, and is optimal for $A$ if $V(A, L)=V_{\mathrm{R}}(A)$.

Example 3.9. Lotteries $L_{1}$ and $L_{2}$ of Example 3.2 are fair for all $A$, and optimal for $A_{2}$ of Example 1 but not for $A_{1}$ or $A_{3}$. Lottery $L_{3}$ is neither fair nor optimal for any $A$ if $n>2$.

Proposition 3.10. Optimal lotteries always exist, and optimal lotteries are always fair.

Proof. Immediate from Theorem 3.7 and Example 3.4(a). 
Proposition 3.11. Let $A \in S_{n, m}$. Then the sets of

(i) fair independent lotteries

and

(ii) optimal independent lotteries

are non-empty closed convex subsets of $S_{m, n}$.

Proof.

(i) The independent lottery $Q$ with entries identically $1 / n$ is fair. To see the set of fair independent lotteries is convex, fix two such lotteries $Q$ and $Q^{\prime}$, and fix $t \in[0,1]$. Then

$$
\begin{aligned}
\left(A\left(t Q+(1-t) Q^{\prime}\right)\right)_{i i} & =\left(t A Q+(1-t) A Q^{\prime}\right)_{i i} \\
& =t(A Q)_{i i}+(1-t)\left(A Q^{\prime}\right)_{i i} \\
& \geq t \cdot \frac{1}{n}+(1-t) \frac{1}{n}=\frac{1}{n},
\end{aligned}
$$

(where the inequality follows from the assumed fairness of $Q$ and $Q^{\prime}$ ), so $t Q+(1-t) Q^{\prime}$ is fair.

The set is closed since if $\left\{Q_{k}\right\}_{k=1}^{\infty}$ are fair and $Q_{k} \rightarrow Q$ (convergence entrywise), then it easily follows that $Q \in S_{m, n}$ and that $Q$ is fair.

(ii) The existence of an optimal independent lottery $Q^{*}$ follows from Theorem 3.7; convexity follows essentially as in (i), replacing $1 / n$ by $V\left(A, Q^{*}\right)$; and closedness also follows aiso as in (i).

If $m=1$, the fair lottery is unique (trivially); conversely, if $m>1$ the set of fair lotteries is never a singleton, as is seen by $L_{1}$ and $L_{2}$ of Example 3.2.

Example 3.12. Let $n=m=2$ and $A=\left(\begin{array}{lll}1 & 0 \\ 0 & 1\end{array}\right)$. Then the optimal lottery is unique, and $V_{\mathrm{R}}(A)=1$.

Remark. For the case $n=2$, Crawford and Heller (1979) give a randomized version of the well-known 'divide-and-choose' solution.

\section{Calculation of optimal lotteries and shares}

The main purpose of this section is to give equivalent characterizations for $V_{\mathrm{R}}$ which enable both the value $V_{\mathrm{R}}(A)$ and an optimal independent lottery $Q$ to be easily calculated. First observe that by Theorem 3.7

$$
V_{\mathrm{R}}(A)=\max \left\{V(A, Q): Q \in S_{m, n}\right\} .
$$

Definition 4.1. For $A \in S_{n, m}$,

The game-theoretic value of $A, V_{\mathrm{G}}(A)$, is 


$$
V_{\mathrm{G}}(A)=\min \left\{\sum_{j=1}^{m} \max _{i \leq n} p_{i} a_{i j}: p_{i} \geq 0, \sum_{i=1}^{n} p_{i}=1\right\}
$$

(Note: This is not the same as the value of a two-person game with payoff matrix $A$.)

The linear-programming value of $A, V_{\mathrm{LP}}(A)$, is the solution of the linear programming problem

$\max z$

s.t.: $\quad z=\sum_{j=1}^{m} a_{i j} q_{j i} \quad i=1, \ldots, n$;

$q_{j i} \geq 0$ for all $i=1, \ldots, n$ and $j=1, \ldots, m$; and

$\sum_{i=1}^{n} q_{j i}=1$ for all $j=1, \ldots, m$.

The value $V_{\mathrm{G}}$ is technically easier to compute than $V_{\mathrm{R}}$, since $V_{\mathrm{G}}$ entails minimizing over an $n$-dimensional probability vector whereas $V_{\mathrm{R}}$ is maximizing over $n \times m$ stochastic matrices. Calculations for both $V_{\mathrm{R}}$ and $V_{\mathrm{G}}$ are, at least superficially, nonlinear; the next theorem, however, shows that they may be reduced to a standard linear programming problem and thus easily solved using LP techniques such as the simplex algorithm.

Theorem 4.2. For all $A \in S_{n, m}$

$$
V_{\mathrm{R}}(A)=V_{\mathrm{G}}(A)=V_{\mathrm{LP}}(A) \text {. }
$$

Proof. Fix $A \in S_{n, m}$. The equality ' $V_{R}(A)=V_{G}(A)$ ' will be proved from a result of Dor (1975) which may be stated as follows:

If $f_{1}, \ldots, f_{n}$ are non-negative Borel functions on $(0, m]$, then

$$
\begin{aligned}
& \min \left\{\int_{0}^{m}\left(\max _{i \leq n} p_{i} f_{i}\right): p_{i} \geq 0, \sum_{i=1}^{n} p_{i}=1\right)=\max \left\{\min _{i} \int_{B_{i}} f_{i}:\left\{B_{i}\right\}_{i}^{n}\right. \\
& \text { is a measurable partition of }(0, m]\} .
\end{aligned}
$$

To see how (1) implies $V_{\mathrm{R}}(A)=V_{\mathrm{G}}(A)$, let $f_{1}, \ldots, f_{n} \geq 0$ be the simple functions on $(0, m)$ given by

$$
f_{i}=a_{i j} \quad \text { on }(j-1, j] \quad \text { for } j=1, \ldots, m, \quad i=1, \ldots, n \text {, }
$$

and for a partition $\left\{B_{i}\right\}_{1}^{n}$ of $(0, m]$, let

$$
q_{j i}=\left(\lambda\left(B_{i}\right)\right)^{-1} \lambda\left(B_{i} \cap(j-1, j]\right),
$$

where $\lambda$ is Lebesque measure. Observe that

$$
Q=\left(q_{j i}\right) \in S_{m, n} .
$$


The conclusion $V_{\mathrm{R}}(A)=V_{\mathrm{G}}(A)$ then follows from (1) and the definitions of $V_{\mathrm{R}}$ and $V_{\mathrm{G}}$ since

$$
\int_{0}^{m} \max _{i \leq n} p_{i} f_{i}=\sum_{j=1}^{m} \int_{j-1}^{j} \max _{i \leq n} p_{i} f_{i}=\sum_{j=1}^{m} \max _{i \leq n} p_{i} a_{i j}
$$

and

$$
\int_{B_{i}} f_{i}=\sum_{j=1}^{m} \int_{B_{i} \cap(j-1, j)} f_{i}=\sum_{j=1}^{m} a_{i j} q_{j i} .
$$

' $V_{\mathrm{R}}(A)=V_{\mathrm{LP}}(A)$ '. This will follow once it is shown that

$$
\begin{aligned}
& \max \left\{\min _{i \leq n}(A Q)_{i i}: Q \in S_{m, n}\right\}=\max \left\{(A Q)_{11}: Q \in S_{m, n}\right. \\
& \text { and } \left.(A Q)_{11}=(A Q)_{i i} \text { for all } i \leq n\right\} .
\end{aligned}
$$

Proof of (2). ' $\geq$ ' is clear since the left-hand-side involves maximizing over a larger set. For ' $\leq$ ', fix $Q^{*} \in S_{m, n}$ so that $V\left(A, Q^{*}\right)=V_{\mathrm{R}}(A)$, and without loss of generality assume $\left(A Q^{*}\right)_{11}=V_{\mathrm{R}}(A)$. If $\left(A Q^{*}\right)_{i i}=V_{\mathrm{R}}(A)$ for all $i \leq n$, the conclusion follows. If $\left(A Q^{*}\right)_{i i}>V_{\mathrm{R}}(A)$, decrease $q_{j i}^{*}$ (i.e., decrease $\left.P(j \rightarrow i)\right)$ for $j$ such that $q_{j i}^{*}>0$ and increase $q_{j i^{\prime}}^{*}$, for those same $j$ and $i^{\prime}$ with $\left(A Q^{*}\right)_{i^{\prime} i^{\prime}}=V_{\mathrm{R}}(A)$, (keeping the row sums equal to 1) in such a way as to decrease $\left(A Q^{*}\right)_{i i}$ to $V_{\mathrm{R}}(A)$, while insuring that the new lottery $\hat{Q}^{*}$ satisfies $\left(A \hat{Q}^{*}\right)_{i i}=V_{\mathrm{R}}(A)$ for all $\hat{i}$ with $\left(A Q^{*}\right)_{i i}=V_{\mathrm{R}}(A)$. Continue until the new lottery $Q$ satisfies $(A \hat{Q})_{11}=\ldots=(A \hat{Q})_{n n}$. This completes the proof of (2).

(Alternatively, the first equality in Theorem 4.2 can be proved using the basic minimax theorem of game theory, and the second equality using linear programming duality.)

Example 4.3. Let $A_{3}$ be as in Example 1. By Theorem 4.2, $V_{\mathrm{R}}\left(A_{3}\right)$ is the solution of

$\max . \quad z$

$$
\begin{array}{ll}
\text { s.t.: } \quad & z=0.7 q_{11}+0.3 q_{21}=0.6 q_{12}+0.4 q_{22} ; \\
& q_{11}, q_{12}, q_{21}, q_{22} \geq 0 ; \\
& q_{11}+q_{12}=q_{21}+q_{22}=1 .
\end{array}
$$

Easy calculations show the optimal lottery $Q=\left(q_{i j}\right)$ is

$$
Q=\left(\begin{array}{cc}
\frac{10}{13} & \frac{3}{13} \\
0 & 1
\end{array}\right), \quad \text { and } \quad A_{3} Q=\left(\begin{array}{cc}
\frac{7}{13} & \frac{6}{13} \\
\frac{6}{13} & \frac{7}{13}
\end{array}\right) \text {, }
$$




$$
V_{\mathrm{R}}\left(A_{3}\right)=\frac{7}{13} .
$$

This last example also shows that lotteries which guarantee that each participant receive at least one object (interesting only in the case $m \geq n$ ) are sometimes strictly sub-optimal; the optimal $Q$ for $A_{3}$ gives both objects to person 2 with probability $\frac{3}{13}$, and it is easily seen that any lottery which guarantees each person an object is strictly sub-optimal.

Recall that by Theorem 3.10, $V_{\mathrm{R}}(A) \geq 1 / n$ for all $A \in S_{n, m}$. The next theorem implies that superfair (i.e., $V_{\mathrm{R}}(A)>1 / n$ ) lotteries exist precisely when not all participants' values agree, and gives sharp bounds for the superfairness based on the 'cooperative' value of the $m$ objects to the $n$ people (i.e., the value obtained if each object is given to a person who values it most, and these values are then summed), and the analogous minimal value, i.e., the value obtained if each object is given to a person who values it least, and these values are summed.

Theorem 4.4. Let $A \in S_{n, m}, \alpha=\sum_{j=1}^{m} \max _{i \leq n}\left\{a_{i j}\right\}$, and $\beta=\sum_{j=1}^{m} \min _{i \leq n}\left\{a_{i j}\right\}$. Then

(i) $V_{\mathrm{R}}(A) \geq(n-\alpha+1)^{-1}$; and

(ii) $V_{\mathrm{R}}(A) \geq(n-1+\beta)^{-1}$,

and these bounds are sharp.

Proof. The conclusion follows, as in the proof of Theorem 4.2, from the following results of Elton, Hill and Kertz (1986) and Hill (1987b): If $\mu_{1}, \ldots, \mu_{n}$ are nonatomic probability measures on $(X, \mathscr{F})$, then $\sup \left\{\min _{i \leq n} \mu_{i}\left(B_{i}\right):\left\{B_{i}\right\}_{i}^{n}\right.$ is a measurable partition of $X\} \geq(n-\alpha+1)^{-1}$, and $\geq(n-1+\beta)^{-1}$, where $\alpha=\sup \left\{\sum_{i=1}^{n}\right.$ $\mu_{i}\left(B_{i}\right):\left\{B_{i}\right\}_{1}^{n}$ is a measurable partition of $\left.X\right\}$ and $\beta=\inf \left\{\sum_{i=1}^{n} \mu_{i}\left(B_{i}\right):\left\{B_{i}\right\}_{1}^{n}\right.$ is a measurable partition of $X\}$.

Corollary 4.5. $V_{\mathrm{R}}(A)>1 / n \Leftrightarrow \operatorname{rank} A>1$.

Proof. Apply Theorem 4.4, observing that $M>1 \Leftrightarrow \operatorname{rank} A>1$. $\square$

(A direct proof of Corollary 4.5 is also possible using redistribution of mass arguments.)

Example 4.6. Let $A_{3}$ be as in Example 1, so $n=2$ and $\alpha=1.1, \beta=0.9$. Then the bound given by Theorem 4.4 (both (i) and (ii)) is $V_{\mathrm{R}}\left(A_{3}\right) \geq \frac{10}{19} \approx 0.5263$. (Recall, from Example 4.3, that $V_{\mathrm{R}}\left(A_{3}\right)=\frac{7}{13} \approx 0.5385$.)

As was stated earlier, the model in this paper assumes each person's true values are known. If one player knows another's values, and that the lotterized solution will be used, he may increase his expected payoff by disguising his preferences, as the next example shows. (One way of guarding against this is to have each player 
submit sealed preferences.)

Example 4.7. Suppose $A_{1}$ represents true preferences, so $V_{\mathrm{R}}\left(A_{1}\right)=\frac{\overline{1}}{13}$. If however, player 2 changes stated preferences to those in $A_{3}$, then player 2 receives object 2 with probability 1 and object 1 with probability $\frac{3}{13}$ for an expected true payoff of $\frac{7}{11}$. (In fact, player 2 should state a preference of $0.7-\varepsilon$ for object 1 and $0.3+\varepsilon$ for object 2 in order to nearly maximize his expected payoff.)

\section{Replication of randomized allocations}

In the previous sections of this paper, it was assumed that there was a one-time distribution of the objects to the participants (either deterministically or randomly). This section addresses the situation when the same objects (or committee assignments, etc.) must be distributed to the same people repeatedly or periodically, and assumes that their values do not change with time.

Definition 5.1. $R(A, L, i)$ is the actual share received by person $i$ (with value matrix $A$ ) under lottery $L$, that is,

$$
R(A, L, i)=\sum_{j=1}^{m} a_{i j} d_{j i}
$$

on the set where $D=\left(d_{j i}\right) \in D_{m, n}$ occurs.

Observe that $R(A, L, i)$ is a uniformly bounded (values in $[0,1])$ random variable whose distribution depends on $A$ and $L$, and that

$$
E(R(A, L, i))=V(A, L, i) \quad \text { for all } i=1, \ldots, n \text {. }
$$

Definition 5.2. Lotteries $L_{1}, L_{2}, \ldots$ are (mutually) independent if $R\left(A, L_{1}, i\right)$, $R\left(A, L_{2}, i\right), \ldots$ are mutually independent for all $i=1, \ldots, n$.

The next theorem says that over time, a repeated use of a one-step optimal lottery yields an average return which is always (i.e., with probability one) better than the average return using any other sequence of lotteries, even ones which are historydependent.

Theorem 5.3. Let $Q_{1}, Q_{2}, \ldots$ be independent copies of an (independent) lottery $Q$ which is optimal for $A$, and let $L_{1}, L_{2}, \ldots$ be a (possibly dependent) sequence of arbitrary lotteries. Then with probability one,

$$
\begin{aligned}
\lim _{j \rightarrow \infty} \min _{i \leq n} \frac{1}{j} \sum_{k=1}^{j} R\left(A, Q_{k}, i\right) & =V_{\mathrm{R}}(A) \\
& \geq \limsup _{j \rightarrow \infty} \min _{i \leq n} \frac{1}{j} \sum_{k=1}^{j} R\left(A, L_{k}, i\right) .
\end{aligned}
$$


Proof. For the equality, observe that $\left\{R\left(A, Q_{k}, i\right)\right\}_{i=1}^{\infty}$ are uniformly bounded i.i.d. random variables with mean $V(A, Q, i)$, so by the strong law of large numbers,

$$
\frac{1}{j} \sum_{k=1}^{j} R\left(A, Q_{k}, i\right) \stackrel{\text { a.s. }}{\rightarrow} V(A, Q, i) \quad \text { for all } i=1, \ldots, n \text {. }
$$

This implies

$$
\lim _{j \rightarrow \infty} \min _{i \leq n} \frac{1}{j} \sum_{k=1}^{j} R\left(A, Q_{k}, i\right) \stackrel{\text { a.s. }}{=} \min _{i \leq n}\{V(A, Q, i)\}=V(A, Q)=V_{\mathrm{R}}(A),
$$

(since $a_{n} \rightarrow a, b_{n} \rightarrow b$ implies $\min \left\{a_{n}, b_{n}\right\} \rightarrow \min \{a, b\}$ ).

For the inequality, consider first the case where the lotteries $L_{1}, L_{2}, \ldots$ are independent. Define

$$
X_{k}(i)=R\left(A, L_{k}, i\right)-V\left(A, L_{k}, i\right),
$$

and observe that for each $i,\left\{X_{k}(i)\right\}_{k=1}^{\infty}$ are uniformly bounded independent meanzero random variables, so

$$
\lim _{j \rightarrow \infty} \frac{1}{j} \sum_{k=1}^{j} X_{k}(i) \stackrel{\text { a.s. }}{=} 0 \quad \text { for all } i \text {. }
$$

This implies

$$
\begin{aligned}
\underset{j \rightarrow \infty}{\limsup } \min _{i \leq n} \frac{1}{j} \sum_{k=1}^{j} R\left(A, L_{k}, i\right) & \stackrel{\text { a.s. }}{=} \limsup _{j \rightarrow \infty} \min _{i \leq n} \frac{1}{j} \sum_{k=1}^{j} V\left(A, L_{k}, i\right) \\
& \leq V_{\mathrm{R}}(A) \text { for all } i=1, \ldots, n .
\end{aligned}
$$

(The inequality follows since for all $j$,

$$
\min _{i \leq n} \frac{1}{j} \sum_{k=1}^{j} V\left(A, L_{k}, i\right)=\min _{i \leq n} V(A, \hat{L}, i)=V(A, \hat{L}) \leq V_{\mathrm{R}}(A),
$$

where $\left.\hat{L}=\left(L_{1}+\ldots+L_{j}\right) / j.\right)$

If $L_{1}, L_{2}, \ldots$ are dependent, define

$$
X_{k}(i)=R\left(A, L_{k}, i\right)-E\left[R\left(A, L_{k}, i\right) \mid \mathscr{F}_{k-1}\right] \quad \text { for each } i=1, \ldots, n,
$$

where $\mathscr{F}_{k}$ is the $\sigma$-algebra generated by $\left\{R\left(A, L_{j}, i\right)\right\}_{j=1}^{k}$. Observe that $\left\{X_{k}(i)\right\}_{k=1}^{\infty}$ is a uniformly bounded martingale difference sequence, so by the martingale strong law of large numbers (Levy, 1937), (3) holds again and consequently

$$
\underset{j \rightarrow \infty}{\lim \sup } \min _{i \leq n} \frac{1}{j} \sum_{k=1}^{j} R\left(A, L_{k}, i\right) \stackrel{\text { a.s. }}{=} \limsup _{j \rightarrow \infty} \min _{i \leq n} \frac{1}{j} \sum_{k=1}^{j} E\left[R\left(A, L_{k}, i\right) \mid \mathscr{F}_{k-1}\right] \text {. }
$$

The conclusion then follows essentially as in the independent case, since

$$
\min _{i \leq n} \frac{1}{j} \sum_{k=1}^{j} E\left[R\left(A, L_{k}, i\right) \mid \mathscr{F}_{k-1}\right]=\min _{i \leq n} \frac{1}{j} V(A, \hat{L}, i),
$$

(where $\hat{L}$ is the $\tilde{F}_{j-1}$-measurable random lottery $L=\left(L_{1}+\ldots+L_{j}\right) / j$ ) and since 
$\min _{i \leq n} V(A, \hat{L}(\omega), i)=V(A, \hat{L}(\omega)) \leq V_{\mathrm{R}}(A)$ for all $\omega$.

The next theorem is similar to Theorem 5.3, but weaker in the sense that the conclusion is for expectations rather that pointwise convergence, and stronger in that the conclusion holds for all $j$, not just in the limit.

Theorem 5.4. Let $\left\{Q_{k}\right\}$ and $\left\{L_{k}\right\}$ be as in Theorem 5.3. Then for all $i \geq 1$,

$$
\min _{i \leq n} E\left[\frac{1}{j} \sum_{k=1}^{j} R\left(A, Q_{k}, i\right)\right]=V_{\mathrm{R}}(A) \geq \min _{i \leq n} E\left[\frac{1}{j} \sum_{k=1}^{j} R\left(A, L_{k}, i\right)\right] .
$$

Proof. The equality follows since the random variables $\left\{R\left(A, Q_{k}, i\right)\right\}_{k=1}^{\infty}$ are i.i.d., so

$$
E\left[\frac{1}{j} \sum_{k=1}^{j} R\left(A, Q_{k}, i\right)\right]=\frac{1}{j} \sum_{k=1}^{j}\left[E\left(R\left(A, Q_{k}, i\right)\right]=V(A, Q, i)\right.
$$

and

$$
\min _{i \leq n} V(A, Q, i)=V(A, Q)=V_{\mathrm{R}}(A)
$$

For the inequality, let $\left\{\hat{L}_{k}\right\}_{k=1}^{j}$ be independent, with $\hat{L}_{k}$ having the same distribution as $L_{k}$ for each $k=1, \ldots, j$. Set $\hat{L}=\left(\hat{L}_{1}+\ldots+\hat{L}_{j}\right) / j \in \mathscr{L}(n, m)$ and observe that

$$
E\left[\frac{1}{j} \sum_{k=1}^{j} R\left(A, L_{k}, i\right)\right]=E[R(A, \hat{L}, i)]=V(A, \hat{L}, i) \quad \text { for all } \quad i \leq n,
$$

so the conclusion follows since

$$
\min _{i \leq n} V(A, \hat{L}, i)=V(A, \hat{L}) \leq V_{\mathrm{R}}(A) \text {. }
$$

The final example points out that the order of the limit and minimization operations in the conclusion of Theorem 5.3 is critical; if one considers the minimum of the limit (superior) average reward rather than the limit of the minimum average reward, then there is always a solution where periodically each person (at different times) may be satisfied he has received the entire estate.

Example 5.5. Let $\left\{D_{i}\right\}_{i=0}^{n-1}$ be the deterministic (and independent) lotteries in which $D_{i}$ assigns all $m$ objects to person $i+1$, and define the sequence of deterministic (and independent) lotteries $Q_{1}, Q_{2}, \ldots$ as follows:

$$
Q_{j}=D_{\hat{k}(\bmod n)}, \quad \text { where } \hat{k}=\min \left\{k: k^{k} \geq j\right\},
$$

(i.e., $Q_{1}=D_{1} ; Q_{j}=D_{2}, j=2,3,4 ; Q_{j}=D_{3}, j=5,6, \ldots, 27$; etc.).

Then it is easy to see that with probability one,

$$
\underset{j \rightarrow \infty}{\limsup } \frac{1}{j} \sum_{k=1}^{j} R\left(A, Q_{k}, i\right)=1 \quad \text { for all } i \text {. }
$$




\section{Acknowledgement}

The authors are grateful to Kevin Phelps for several conversations, to Jan van der Wal for suggesting the use of randomized allocations, and to the referee for several suggestions and corrections.

\section{References}

V. Crawford and W. Heller, Fair division with indivisible commodities, J. Econom. Theory 21 (1979) $10-27$.

L. Dor, On projections in $L_{1}$, Ann. Math. 102 (1975) 463-474.

L. Dubins, Group decision devices, Amer. Math. Monthly 84 (1977) 350-356.

J. Elton, T. Hill, and R. Kertz, Optimal partitioning inequalities for nonatomic probability measures, Trans. Amer. Math. Soc. 296 (1986) 703-725.

M.R. Garey and D.S. Johnson, Computers and Intractability (Freeman, San Francisco, 1979).

T. Hill, Partitioning general probability measures, Ann. Probab. 15 (1987a) 804-813.

T. Hill, A sharp partitioning-inequality for nonatomic probability measures based on the mass of the infimum of the measures, Probab. Theory and Related Fields 75 (1987b) 143-147.

E. Kalai, Solutions of the bargaining problem, in: L. Hurwicz, D. Smeidler and H. Sonnenshein, eds., Social Goals and Organization, Essays in memory of Elisha Pazner (Cambridge University Press, 1985) pp. 77-105.

P. Levy, Theorie de l'addition des variables aléatories (Gauthier-Villars, Paris, 1937).

H. Steinhaus, The problem of fair division, Econometrica 16 (1948) 101-104.

H. Steinhaus, Sur la division pragmatique, Econometrica (supplement) 17 (1949) 315-319.

H. Steinhaus, Mathematical Snapshots (Oxford University Press, New York, 1960). 